\title{
A PROPOSED TECHNOLOGY SOLUTION FOR ENHANCING ORDER PICKING IN WAREHOUSES AND DISTRIBUTION CENTERS BASED ON A GAMIFIED AUGMENTED REALITY APPLICATION
}

\author{
George Plakas, Eleni Aretoulaki, Stavros T. Ponis, Konstantinos Agalianos \\ and Theodoros Nikolaos Maroutas \\ School of Mechanical Engineering, National Technical University Athens \\ Heroon Polytechniou 9, Zografos 15780, Athens, Greece
}

\begin{abstract}
Industry 4.0, since the first introduction of the term back in 2011, has gained significant traction and nowadays it is -almost unanimously- considered as the main technology driver, leading supply chain in the new era of faster, flexible and more intelligent processes and reduced operational, planning and management costs. One of the most prominent technologies in the Industry 4.0's arsenal is Augmented Reality (AR), which utilizes the capabilities of computer-generated display, sound, text and effects to enhance the user's real-world experience, through wearable equipment. In logistics environments, the use of AR to support workers in their everyday order picking tasks is currently a business reality with growing number of applications and significant scaling potential. However, in this new business reality, despite the efforts made to support workers, order picking still stands out as the most labor intensive, prone to error and expensive process in the modern warehouse. These characteristics indisputably put significant strain to pickers creating both fatigue, feelings of monotony and dissatisfaction usually leading to employee underperformance and demotivation. This paper proposes a technology solution that introduces AR in the picking process to efficiently support pickers in their everyday tasks, while at the same time evaluates the potential of enhancing AR with gamification elements as a tool for increasing motivation, positively influence job satisfaction and behavior and subsequently increase performance and productivity.
\end{abstract}

\section{KEYWORDS}

Augmented Reality, Gamification, Order Picking, Warehouses, Smart Glasses, Logistics 4.0

\section{INTRODUCTION}

Logistics state-of-the-art has evolved in recent years, since warehouses and distribution centers are facing challenges, such as the need of faster and more efficient operations and reduced inventory maintenance. Major factor for these challenges is the global development of e-commerce and upward demand for personalized products. The most labor intensive task in warehousing and supply chain management is the order picking process (De Koster, 2007). It is estimated that $75 \%$ of the tasks in a warehouse are conducted by warehouse workers and more than 50\% (Elbert and Sarnow, 2019) of the operating cost of the warehouse derives from order picking procedures. While this process is necessary, its traditional approach is expensive, prone to errors and with high labor intensity. The most important measurable efficiency indicators of order picking are time and accuracy (Reif and Günthner, 2009; Reif et al., 2010). Time determines the productivity of the warehouse's workforce and affects the efficiency of the entire supply chain. Lack or accuracy and errors on the other hand, have a severe impact on the warehousing quality, as they can lead to significant financial consequences. The three most common errors in order picking are: a) wrong item collection, b) collection of items in the wrong quantity and c) omission of items.

One of the Industry 4.0 technologies, that are tested to improve the intralogistics performance, is Augmented Reality (AR), which refers to the addition of computer-generated visualizations, to the real environment and is mainly displayed through smart wearable systems. The AR purpose is adding objects to a real environment, but also hiding objects from it, in real time (Azuma et al., 2001). In the near future, warehouse 
workers will use their smartphones, wearables and other AR and VR devices to join the IoT environment and increase human-to-machine interaction (Efthymiou and Ponis, 2019). As a matter of fact, AR is able to support warehousing, providing the possibility of an interactive work with fewer errors (Cirulis and Ginters, 2013) and as result rendering pickers' daily tasks simpler, enjoyable and less stressful. Visual content provided by a wearable system in picker's field of view is able to reduce the time between information's perception and action (Wang and Dunston, 2006). Thus, in the specific case of order picking, labor effort and mental fatigue may be reduced and efficiency subsequently increased. Moreover, a wearable system sets both picker's hands free, facilitating the productive part of his/her work (picking). Evidently, the most important task that can be improved by an AR system is the navigation. According to Schwerdtfeger et al. (2011), order picking involves two phases of navigation. In the first phase (course navigation), the picker has to walk to the right aisle and rack. In the second phase (fine navigation), the picker stands in front of the right rack and has to locate the correct shelf, bin or box, to complete the collection. The second phase is deemed the most critical, especially for distribution centers providing an extensive variety of small items. With the utilization of an AR navigation system, it is possible to present the employee with visual navigation aids, displaying the exact position of the targeted item to pick. As a result, the time for finding and collecting products will be substantially reduced and potential errors may be prevented, leading to a more productive order picking process.

The technique of using AR as an aid-providing subsystem of the order picking process has been named Pick-by-Vision. The first appearance of this term in modern literature is by Reif and Walch (2008). A search in the literature brought to the foreground some existing studies focusing on improving the way navigation of a picker through the warehouse's aisles is carried out and searching for the optimal way to improve fine navigation. Despite the fact that the efforts of AR researchers in this field are still young, there are already commercial AR systems for order picking (Guo et al., 2014) and systems used by high-profile players in the supply chain industry (DHL Logistics, 2019).

Nevertheless, in spite of its indisputable merits, AR technology is unable to countermand the fact that order picking is an inherently intensive and repetitive manual process, leading to fatigue, demotivation (Hense et al., 2013) and consequently, decreased performance in the long run, increasing operational costs for the supply chain overall (De Koster et al., 2007). So, it goes without saying that the enhancement of motivation is a primary factor in ameliorating the performance of order picking (van den Berg, 2017). Gamification, i.e. "the use of game design elements in non-game contexts" (Deterding, 2011) has proved to be able to effectively serve this purpose, by using game elements to meet various psychological needs, such competence, autonomy and social relatedness, with the aim of motivating workers, influencing their behavior and as a result, improving their productivity. The proposed solution presented in this paper appraises the potential of introducing AR and game elements into the order picking process of modern distribution centers, with a view to assisting pickers' daily tasks, increasing their motivation and hence, their performance.

\section{THE PROPOSED SOLUTION}

The proposed technology solution involves the development of a system aspiring to support man-to-goods order picking within warehouses and distribution centers. The solution aims to combine the functionalities of Augmented Reality and the techniques of Gamification and its final product will be able to be fully integrated into any existing Warehouse Management System (WMS). The development of the system consists of a set of two integrated modules (i.e. AR picking module and Gamification module) which are connected through appropriate APIs to the existing WMS utilized by the warehouse. The existing WMS is a key component of the final product, as it transfers data to both modules and receives data from the AR Picking module. The design and development of a comprehensive solution that can be configured to meet all possible needs and address various scenarios that may occur during the order picking process, within any warehousing establishment, is of great importance for the authors.

The AR Picking module is responsible for providing the picker in his/her eye level with the necessary information for collecting the item (ID, position, quantity) and subsequently navigating him/her through an imaging feature, able to solve any course and fine navigation issues, utilizing three-dimensional arrows and shapes to highlight the right isle or self, according to the data provided by the WMS. During standard AR-enhanced collection, the picker will be able to complete his/her assignment by a) understanding the order received from WMS b) following the route provided by the imaging feature, c) collecting the item from the 
right shelf and d) informing the WMS about the finalized collection, through one of the smart glasses' functionalities (buttons, microphone, camera etc.). The process will be repeated until the list is over and it is significant that the picker's hands are both free during this process, thanks to the wearable equipment.

Evidently, the smart wearable system is the most important part of the AR Picking module and the choice of the appropriate visualization device would affect the successful implementation of the entire solution. The basic challenge for the smart glasses is the fact that the employees are required to wear them throughout their shift, so they should be lightweight and ergonomically designed. Also, the smart glasses' screen should not interfere with picker's field of view towards the real warehouse environment, for safety reasons. At the same time, the operating time of the battery has to be sufficient and need no recharging until the end the picker's shift. The authors believe that all of these are covered sufficiently by an industrial product, VUZIX M300XL (Vuzix, 2020).

On the other hand, the Gamification module will be responsible for providing a new working experience, by enabling managers to create a gaming environment (scenarios) for the employees to compete in. The gamification mechanism will operate around this set of work-related competition scenarios among the pickers and the WMS will feed data into the Process Center of the Gamification module to enable the attainment of goals. The authors believe that the best way to enhance the order picking process is to create scenarios based on the most important measurable efficiency indicators of order picking as they were mentioned in the introduction, which are time and accuracy. Taking this into account, every correct collection will add points to picker's gamification profile and every error (collection of wrong item or wrong quantity of an item collected) will have negative impact to the profile, subtracting multiple points. Last but not least, a quick collection will be rewarded by offering bonus points to the picker. The warehouse managers can take advantage of these basic elements to build different scenarios, either based on personal goals for each one of their employees or based on weekly or monthly leaderboards to reward exclusively the best-performing pickers in a period of time. Leaderboards and rankings announcing performance-related information will be displayed to warehouse managers, supervisors and pickers, by the Report Center of the Gamification module, through a web reporting tool, which could be also displayed via a public screen within the warehouse. The authors believe that a gamified approach is a solution that can potentially boost pickers' productivity and performance and combined with the game-related interface offered by the AR Application, the system is expected to meet high acceptance levels. The entire system has to be exploited as a whole, in order to upgrade the work experience within the warehouse to a flexible, smart and playful process for the benefit of the entire supply chain. A schematic of the proposed solution is presented in Figure 1.

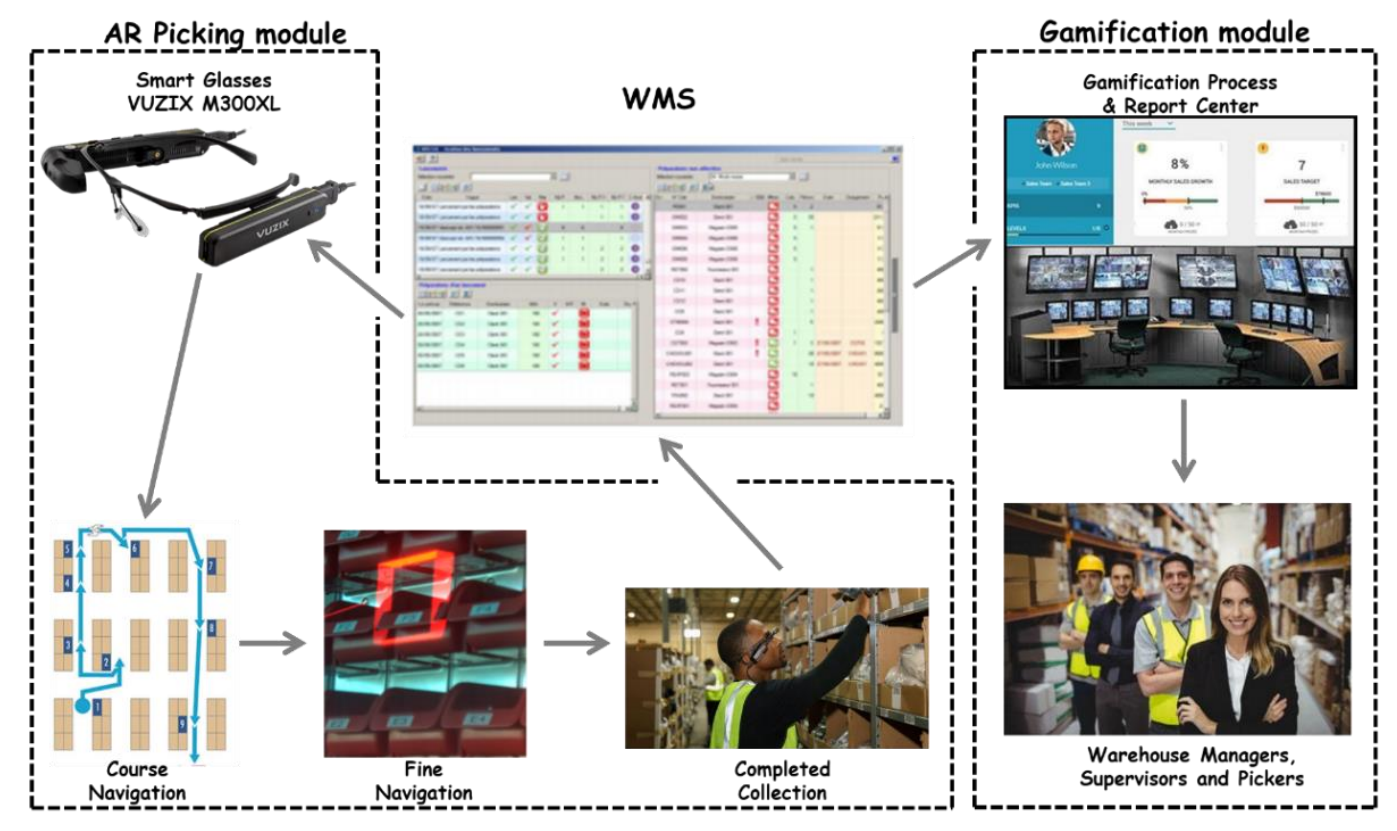

Figure 1. The proposed technology solution 
The authors are currently in the phase of assessing the technical components and the overall viability of the system, while at the same time identifying possible ways for design improvements. Their future objective is to produce a small-scale pilot-tested Proof of Concept (POC), including:

- An ergonomic coupling of the system's hardware components (VUZIX M300XL, battery, warehouse helmet).

- A generic and fully customizable man-to-goods AR order picking application, integrated in the VUZIX M300XL android software.

- A gamification application for setting all the competition scenarios between pickers and calculating results according the WMS-provided data.

- A fully integrated web reporting tool informing the stakeholders of the system (warehouse managers, supervisors and pickers) about the results of the competitions.

- APIs for connection with the existing WMS (one API for each module).

\section{CONCLUSION}

The proposed solution will provide warehouse managers with innovative software and its final product will be seamlessly integrated with existing warehouse management systems in a WMS close to agnostic fashion, in order to effectively support the order picking process. Hardware and software capabilities of all AR applications still require improvements, but the technology has already demonstrated its potential and left many promises for the future, as a new way of supporting the intralogistics workforce. Through the AR Picking module, the authors focus on making the order picking process faster, with fewer errors and more ergonomic for the employees. Providing information on pickers' and items' real-time position is able to present the former with navigation instructions, drastically reducing time, both on course and fine navigation. Furthermore, through the Gamification module, the authors try to achieve greater employee engagement and offer incentives to increase productivity, which combined with AR's ability to dispense pickers from needing to use their hands, will transform their daily tasks into an enjoyable routine. All in all, research on the implementation of AR and Gamification in order picking is still in progress and the authors plan to further explore its potential in the future, by examining the possibility of adding live Gamification statistics to the glasses' screen, while taking into account that such a decision could be conducive to stress and countermanding the benefits of Gamification.

\section{ACKNOWLEDGEMENT}

The present work is co-funded by the European Union and Greek national funds through the Operational Program "Competitiveness, Entrepreneurship and Innovation" (EPAnEK), under the call "RESEARCH-CREATE-INNOVATE" (project code: T1EDK-01168 \& acronym: SMARTFLEX Warehouse).

\section{REFERENCES}

Azuma R. et al, 2001. Recent advances in augmented reality. IEEE computer graphics and applications, 21(6), pp.34-47. Cirulis A. and Ginters E., 2013. Augmented reality in logistics. Procedia Computer Science, 26(11), pp.14-20.

De Koster R. et al., 2007. Design and control of warehouse order picking: A literature review. European journal of operational research, 182(2), pp.481-501.

Deterding S. et al., 2011, September. From game design elements to gamefulness: defining" gamification". In Proceedings of the 15th international academic MindTrek conference: Envisioning future media environments (pp. 9-15).

DHL Logistics, 2019. DHL SUPPLY CHAIN DEPLOYS LATEST VERSION OF SMART GLASSES WORLDWIDE. Available at: <https://www.dhl.com/global-en/home/press/press-archive/2019/dhl-supply-chain-deploys-latestversion-of-smart-glasses-worldwide.html> [Accessed 10 June 2020].

Efthymiou, O.K. and Ponis, S.T., 2019. Current Status of Industry 4.0 in Material Handling Automation and In-house Logistics. International Journal of Industrial and Manufacturing Engineering, 13(10), pp.1370-1374. 
Elbert R. and Sarnow T., 2019, February. Augmented Reality in Order Picking-Boon and Bane of Information (Over-) Availability. In International Conference on Intelligent Human Systems Integration (pp. 400-406). Springer, Cham.

Guo A. et al., 2014, September. A comparison of order picking assisted by head-up display (HUD), cart-mounted display (CMD), light, and paper pick list. In Proceedings of the 2014 ACM International Symposium on Wearable Computers (pp. 71-78).

Hense J. et al., 2013, June. Using gamification to enhance staff motivation in logistics. In International Simulation and Gaming Association Conference (pp. 206-213). Springer, Cham.

Reif R. and Walch D., 2008. Augmented \& Virtual Reality applications in the field of logistics. The Visual Computer, 24(11), pp.987-994.

Reif R. et al., 2010, March. Evaluation of an augmented reality supported picking system under practical conditions. In Computer Graphics Forum (Vol. 29, No. 1, pp. 2-12). Oxford, UK: Blackwell Publishing Ltd.

Reif, R. and Günthner, W.A., 2009. Pick-by-vision: augmented reality supported order picking. The Visual Computer, 25(5-7), pp.461-467.

Schwerdtfeger B. Et al., 2011. Pick-by-vision: there is something to pick at the end of the augmented tunnel. Virtual reality, 15(2-3), pp.213-223.

van den Berg, T., 2017. Gamification to enhance motivation in a real world order picking environment of an online grocer: A case study at Picnic.

Vuzix, 2020. Vuzix M300XL | Industry AR Solutions. [online] Available at: <https://www.vuzix.com/products/m300xlsmart-glasses> [Accessed 7 June 2020].

Wang X. and Dunston P.S., 2006. Compatibility issues in Augmented Reality systems for AEC: An experimental prototype study. Automation in construction, 15(3), pp.314-326. 